TRANSACTIONS OF THE

AMERICAN MATHEMATICAL SOCIETY

Volume 350, Number 9, September 1998, Pages 3483-3504

S $0002-9947(98) 02247-8$

\title{
CONNECTED FINITE LOOP SPACES WITH MAXIMAL TORI
}

\author{
J. M. MØLLER AND D. NOTBOHM
}

\begin{abstract}
Finite loop spaces are a generalization of compact Lie groups. However, they do not enjoy all of the nice properties of compact Lie groups. For example, having a maximal torus is a quite distinguished property. Actually, an old conjecture, due to Wilkerson, says that every connected finite loop space with a maximal torus is equivalent to a compact connected Lie group. We give some more evidence for this conjecture by showing that the associated action of the Weyl group on the maximal torus always represents the Weyl group as a crystallographic group. We also develop the notion of normalizers of maximal tori for connected finite loop spaces, and prove for a large class of connected finite loop spaces that a connected finite loop space with maximal torus is equivalent to a compact connected Lie group if it has the right normalizer of the maximal torus. Actually, in the cases under consideration the information about the Weyl group is sufficient to give the answer. All this is done by first studying the analogous local problems.
\end{abstract}

\section{INTRODUCTION}

A loop space $L:=(L, B L, e)$ is a triple consisting of two spaces $L$ and $B L$, which is pointed, and an equivalence $e: \Omega B L \rightarrow L$ between the loop space of $B L$ and $L$. The space $B L$ is called the classifying space of $L$. A loop space $L$ is called finite if $L$ is $\mathbb{Z}$-finite, i.e. the integral cohomology $H^{*}(L ; \mathbb{Z})$ is a finitely generated graded $\mathbb{Z}$-module. A finite loop space $L$ is called connected if $L$ is a connected space. For a compact Lie group $G$ the triple $(G, B G, e)$ is a finite loop space, where $e: \Omega B G \rightarrow G$ is the obvious equivalence.

Following an old idea of Rector [19], several notions of (Lie) group theory can be given in homotopy theoretic terms by means of classifying spaces (e.g. see [19], [18], [5], [6], [13]). For example, a homomorphism $f: L \rightarrow M$ of finite loop spaces is a pointed map $B f: B L \rightarrow B M$. The homotopy fiber of $B f$ is denoted by $M / L$. A homomorphism $f: L \rightarrow M$ is an isomorphism if $B f$ is a homotopy equivalence. In particular, we are interested in maximal tori and the associated Weyl groups of connected finite loop spaces. We will use the following definition:

1.1 Definition. Let $L$ be a connected finite loop space. A maximal torus of $L$ is a homomorphism $f: T \rightarrow L$ from a torus $T$ into $L$ which satisfies the following two conditions:

1. The homogeneous space $L / T$ is $\mathbb{Z}$-finite.

Received by the editors July 11, 1995.

1991 Mathematics Subject Classification. Primary 55P35, 55R35.

Key words and phrases. Finite loop space, $p$-compact group, classifying space, maximal torus, normalizer, Weyl group, covering.

(C)1998 American Mathematical Society 
2. The rank $r k(L)$ of $L$ equals the rank $r k(T)$ of $T$. The rank is defined to be the transcendence degree of the polynomial ring $H^{*}(B L ; \mathbb{Q})$ over $\mathbb{Q}$.

The Weyl monoid $W_{L}:=W_{L}(T)$ of a maximal torus $f: T \rightarrow L$ is the monoid

$$
W_{L}(T):=\{[w: B T \rightarrow B T] \mid B f \circ w \simeq B f\}
$$

of all homotopy classes of self maps of $B T$ stabilizing the structure map $B f: B T \rightarrow$ $B L$.

The classical maximal torus $T_{G} \rightarrow G$ of a compact connected Lie group $G$ passes to a fibration $G / T_{G} \rightarrow B T \rightarrow B G$, and an element $w \in W_{G}$ of the Weyl group $W_{G}$ of $G$ give rise to a homotopy commutative diagram

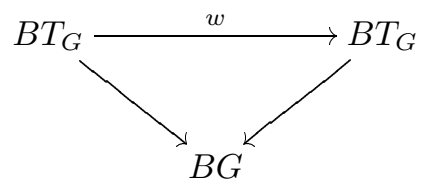

Hence, the above definitions are the extract of the classical situation in terms of classifying spaces. We also show that every two maximal tori of a finite loop space are conjugate and that the definition of the Weyl group does not really depend on the chosen maximal torus (Proposition 3.5).

Usually, when talking about finite loop spaces, the $\mathbb{Z}$-finiteness condition is replaced by the condition of being homotopy equivalent to a finite $C W$-complex, which is a slightly stronger condition. For our purpose $\mathbb{Z}$-finiteness is sufficient.

Having a maximal torus is a quite distinguished property for finite loop spaces. It is well known that there exist finite loop spaces which don't have a maximal torus. Examples may be found in [19] or [18]. Actually, an old conjecture, which we could trace back to [20], says that a connected finite loop space a with maximal torus is isomorphic to a compact connected Lie group (as loop space). We want to give some more evidence for this conjecture. An integral representation $W \rightarrow G l(n, \mathbb{Z})$ of a finite group $W$ is called crystallographic, if the associated rational representation $W \rightarrow G l(n, \mathbb{Q})$ represents $W$ as a reflection group. We define $L T_{L}:=H_{2}\left(B T_{L}, \mathbb{Z}\right)$ and $L^{*} T_{L}:=H^{2}\left(B T_{L}, \mathbb{Z}\right)$.

1.2 Theorem. Let $L$ be a finite loop space with maximal torus $T \rightarrow L$. Then the following hold:

1. The Weyl monoid $W_{L}$ is a group.

2. The induced action of $W_{L}$ on $L^{*} T_{L}$ represents $W_{L}$ as a crystallographic group.

3. We have $H^{*}(B L ; \mathbb{Q}) \cong H^{*}(B T ; \mathbb{Q})^{W_{L}}$.

The representation $W_{L} \rightarrow G l\left(L^{*} T_{L}\right)$ is called the associated representation.

Examples of crystallographic representations $W \rightarrow G l(n, \mathbb{Z})$ are given by actions of Weyl groups of compact connected Lie groups on the 2-dimensional homology or cohomology of the classifying space of the maximal torus. We say that a connected finite loop space $L$ with maximal torus $T_{L} \rightarrow L$ has the same Weyl group type as a compact connected Lie group $G$ if $r k(L)=r k(G)=: n$ and if the two associated representations $W_{L}, W_{G} \rightarrow G l(n, \mathbb{Z})$ are conjugate. The following question is a weaker and slightly different form of the above mentioned conjecture. Let $L$ be a connected finite loop space with the same Weyl group type as the compact connected Lie group $G$. Does this imply that $L$ and $G$ are isomorphic as loop spaces? This conjecture can't be true. The compact connected Lie groups $S O(2 n+1)$ 
and $S p(n)$ are not isomorphic as loop spaces, because the existence of a homotopy equivalence $B S O(2 n+1) \simeq B S p(n)$ would imply that $S O(2 n+1)$ and $S p(n)$ were isomorphic as Lie groups [16]. But on the other hand they have the same Weyl group type.

The right invariant to distinguish the isomorphism types of connected finite loop spaces with maximal tori should be given by the normalizer of the maximal torus. Some evidence for this conjecture comes from [16], where it is proved that two compact connected Lie groups $G$ and $H$ are isomorphic (as Lie groups) if and only if the classifying spaces are homotopy equivalent if and only if the classifying spaces of the two normalizers of the maximal tori are equivalent if and only if the normalizers itself are isomorphic (as Lie groups) for the loop spaces associated with compact connected Lie groups.

1.3 Definition. Let $L$ be a connected finite loop space with maximal torus $T_{L} \rightarrow$ $L$, The normalizer of $T_{L}$ is a monomorphism $N\left(T_{L}\right) \rightarrow L$ of finite loop spaces such that $N\left(T_{L}\right)$ fits up to homotopy into a fibration

$$
B T_{L} \rightarrow B N\left(T_{L}\right) \rightarrow B W_{L}
$$

and such that

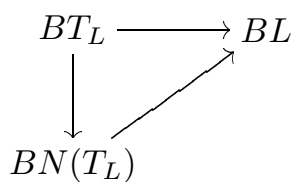

commutes up to homotopy.

1.4 Proposition. Let $L$ be a connected finite loop space with maximal torus $T_{L} \rightarrow$ $L$. Then there exists a normalizer $N\left(T_{L}\right)$ of the maximal torus, and $N\left(T_{L}\right)$ is isomorphic to a uniquely determined compact Lie group as loop space.

In order to proceed with the maximal torus conjecture we first want to fix some notation about compact connected Lie groups. For every compact connected Lie group $G$ there exists a finite covering $K \rightarrow G_{s} \times T \rightarrow G$ of compact Lie groups, where $G_{s}$ is simply connected, where $T$ is a torus, and where $K \subset G_{s} \times T$ is a finite central subgroup.

1.5 Definition. Let $G$ be a compact connected Lie group.

1. $G$ satisfies condition $\left(T_{p}\right)$ if $H^{*}(G ; \mathbb{Z})$ or, equivalently, $H^{*}(B G ; \mathbb{Z})$ is $p$-torsion free.

2. $G$ satisfies condition $\left(I_{p}\right)$ if $H^{*}\left(B G ; \mathbb{F}_{p}\right) \cong H^{*}\left(B T_{G} ; \mathbb{F}_{p}\right)^{W_{G}}$.

3. $G$ satisfies condition $\left(C_{p}\right)$ if $G$ satisfies $\left(T_{p}\right)$ and $G_{s}$ the condition $\left(I_{p}\right)$.

In [15] a compact connected Lie group $G$ is called $p$-convenient if it satisfies $\left(C_{p}\right)$. This condition was used there to prove homotopy uniqueness results for classifying spaces of compact connected Lie groups. We will apply similar methods here to classify the isomorphism type of compact connected Lie groups as loop spaces using the normalizer. Each of the above three conditions is weaker than the following one. But for an odd prime all three conditions are equivalent [15]. We also notice that $U(2)$ satisfies $\left(I_{2}\right)$ but not $\left(C_{2}\right)$, because $S U(2)$ does not satisfy $\left(T_{2}\right)$. A complete list of simple simply connected compact Lie groups satisfying $\left(C_{p}\right)$ may be found in $[15$, Chapter 1]. 
1.6 Theorem. Let $G$ be a compact connected Lie group satisfying conditions $\left(C_{2}\right)$ and $\left(T_{p}\right)$ for all primes. Let $L$ be a connected finite loop space with maximal torus $T_{L} \rightarrow L$. Then the following statements are equivalent:

1. L has the same Weyl group type as $G$.

2. $N\left(T_{G}\right)$ and $N\left(T_{L}\right)$ are isomorphic as Lie groups.

3. $B N\left(T_{G}\right)$ and $B N\left(T_{L}\right)$ are homotopy equivalent.

4. $B G$ and $B L$ are homotopy equivalent.

1.7 Remark. There is an interesting observation related to Proposition 1.4. For a given extension $N(T)$ of an operation of a Weyl group $W$ on a maximal torus $T$ it is not known if there exists a connected finite loop space with these data. For example, there are two extensions of the permutation representation of the symmetric group $\Sigma_{n} \cong W_{U(n)}$ on $\left(S^{1}\right)^{n}=T_{U(n)}$, namely the semidirect product and a nontrivial extension. But Theorem 1.6 says that only the semidirect product can occur as the normalizer of a maximal torus of a connected finite loop space.

To study finite loop spaces, Dwyer and Wilkerson introduced the notion of $p$ compact groups in their influential paper [5]. A loop space $X$ is a $p$-compact group if the classifying space $B X$ is $p$-complete and if $X$ is $\mathbb{F}_{p}$-finite, i.e. $H^{*}\left(X ; \mathbb{F}_{p}\right)$ is finite [5]. The completion of a connected finite loop space always gives a $p$-compact group. For nonconnected finite loop spaces one has to assume that the group of the components is a finite $p$-group.

The $p$-compact groups behave very much like compact Lie groups. For example, a $p$-compact group always has a maximal torus in the sense of [5]. Dwyer and Wilkerson also constructed a normalizer $N\left(T_{X}\right)$ of the maximal torus $T_{X} \rightarrow X$ which satisfies the analogous properties as in Lemma 1.3 of [5] (see also Section 2). The classifying space $B N\left(T_{X}\right)$ fits into a fibration $B T_{X} \rightarrow B N\left(T_{X}\right) \rightarrow B W_{X}$. In particular, $B N\left(T_{X}\right)$ is a fiberwise complete space with respect to this fibration. For details see Section 2.

For a fibration $F \rightarrow E \rightarrow B$ we denote by $E_{p}^{\circ}$ the fiberwise $p$-adic completion [4].

1.8 Proposition. Let $L$ be a connected finite loop space.

1. For any prime $p$ the triple $\left(L_{p}^{\wedge}, B L_{p}^{\wedge}, e_{p}^{\wedge}\right)$ is a p-compact group.

2. If $T \rightarrow L$ is a maximal torus of $L$, then completion gives rise to a maximal torus $T_{p}^{\wedge} \rightarrow L_{p}^{\wedge}$ and induces a natural isomorphism $W_{L} \cong W_{L_{p}}$ and a homotopy equivalence $B N\left(T_{L}\right)_{p}^{\circ} \simeq B N\left(T_{L p} \wedge\right)$.

The Weyl group $W_{X}$ of a $p$-compact group $X$ acts on $L^{*} T_{X p} \wedge:=H^{*}\left(B T_{X} ; \mathbb{Z}_{p}^{\wedge}\right)$ and gives rise to a representation $W_{X} \rightarrow G l\left(L^{*} T_{X} \wedge\right)$. By the above lemma the completion of a connected finite loop space with maximal torus gives a connected $p$-compact group with maximal torus. We say that a connected $p$-compact group $X$ has the $p$-adic Weyl group type of a compact connected Lie group $G$ if $r k(X)=r k(G)=: n$ and if the associated representations $W_{X}, W_{G} \rightarrow G l\left(n, \mathbb{Z}_{p}^{\wedge}\right)$ are conjugate.

The next statement is the completed version of Theorem 1.6.

1.9 Theorem. Let $G$ be a compact connected Lie group satisfying condition $\left(T_{p}\right)$ if $p$ is odd or $\left(C_{2}\right)$ if $p=2$. Let $X$ be a connected $p$-compact group. Then the following conditions are equivalent:

1. $X$ has the same $p$-adic Weyl group type as $G$. 
2. $B N\left(T_{G}\right)_{p}^{\circ}$ and $B N\left(T_{X}\right)$ are homotopy equivalent.

3. $B G_{p}^{\wedge}$ and $B X$ are homotopy equivalent, i.e. $G_{p}^{\wedge}$ and $X$ are isomorphic $p-$ compact groups.

Using the $p$-adic result, we are able to prove Theorem 1.6.

Proof of Theorem 1.6. Let us assume that (1) is satisfied, i.e. $L$ and $G$ have the same Weyl group type. The Weyl group type of a connected finite loop space determines the $p$-adic Weyl group type of the associated connected $p$-compact group. By Theorem 1.9 this implies that $B L_{p}^{\wedge} \simeq B G_{p}^{\wedge}$ for every prime $p$. Moreover, we also have a rational equivalence, because both spaces are rationally a product of Eilenberg-Mac Lane spaces, and because $H^{*}(B L ; \mathbb{Q}) \cong H^{*}\left(B T_{G} ; \mathbb{Q}\right)^{W_{G}} \cong$ $H^{*}(B G ; \mathbb{Q})$ (Theorem 1.2). That is to say that $B L$ and $B G$ have the same adic genus. By assumption $L$ has a maximal torus, which implies that there exists a compact connected Lie group $H$ such that $B L \simeq B H$ [14]. Because the two associated representations of the Weyl groups are isomorphic, we also have $K(B G) \cong$ $K\left(B T_{G}\right)^{W_{G}} \cong K\left(B T_{H}\right)^{W_{H}} \cong K(B H)$ as $\lambda$-rings. The compact connected Lie group $G$ satisfies condition $\left(C_{2}\right)$. Because every quotient of a Spin-group has 2torsion in the integral cohomology, the finite cover $G_{s} \times T$ of $G$ cannot contain a factor isomorphic to $\operatorname{Spin}(n)$ for some $n$. In this situation we can apply $[15,1.6]$ to conclude that $B X \simeq B G$. This is condition (4).

If $B L \simeq B G$, then $B N\left(T_{G}\right)_{p}^{\circ} \simeq B N\left(T_{L}\right)_{p}^{\circ}$ for any prime (Theorem 1.9). This is really an equivalence of the associated fibrations, and implies therefore that $B N\left(T_{G}\right) \simeq B N\left(T_{L}\right)$ [16, proof of 3.6], which is condition (3). By [16, Theorem A], a homotopy equivalence $B N\left(T_{G}\right) \simeq B N\left(T_{L}\right)$ establishes an isomorphism $N\left(T_{G}\right) \cong$ $N\left(T_{L}\right)$ as compact Lie groups, which is condition (2).

If $N\left(T_{G}\right) \cong N\left(T_{L}\right)$ as compact Lie groups, then it is obvious that $G$ and $L$ have the same Weyl group type. This completes a circle of implications.

The paper is organized as follows: Section 2 is devoted to $p$-compact groups. From [5] we will recall how notions of (Lie) group theory translate to $p$-compact groups. We also recall necessary results from [6] and [13]. In Section 3 we discuss the (integral) Weyl group of finite loop spaces and prove Theorem 1.2, Proposition 1.4 and Proposition 1.8. Section 4 provides some more results about $p$-compact groups necessary for the proof of Theorem 1.9. In particular, we study homotopy classes of liftings of maps into a connected $p$-compact group to the maximal torus. The last three sections are devoted to the proof of Theorem 1.9, which is split into several cases. First we discuss uniqueness results for products of unitary groups (Section 5), then for simply connected compact Lie groups (Section 6), and finally in the general case (Section 7). Section 7 also contains a proof of Theorem 1.9.

Completion and localization are always meant in the sense of Bousfield and Kan $[4]$.

\section{2. $p$-COMPACT GROUPS}

In this section we recall the basic notions and the fundamental results about $p$ compact groups from [5]. Most of the notions are motivated by classical Lie group theory and by passing to classifying spaces. To keep things short, and because the analogy to compact Lie groups is discussed in [5], [6] and [13], we omit motivations. 
2.1 Homomorphisms, monomorphisms, isomorphisms, subgroups and exact sequences. A homomorphism $f: Y \rightarrow X$ of $p$-compact groups is a pointed map $B Y \rightarrow B X$. The map $f$ is called an isomorphism if $B f: B Y \rightarrow B X$ is an equivalence. A sequence $X \stackrel{f}{\rightarrow} Y \stackrel{g}{\rightarrow} Z$ of $p$-compact groups is short exact if the associated sequence $B X \stackrel{B f}{\longrightarrow} B Y \stackrel{B g}{\longrightarrow} B Z$ is a fibration up to homotopy. A monomorphism of $p$-compact groups is a map $B X \rightarrow B Y$ whose homotopy fiber, denoted by $Y / X$, is $\mathbb{F}_{p}$-finite. A subgroup $Y \rightarrow X$ of a $p$-compact group $X$ is a monomorphism of $p$-compact groups.

$2.2 p$-compact toral groups. A $p$-compact toral group $P$ is a $p$-compact group $P$ fitting into a short exact sequence $T \rightarrow P \rightarrow \pi$, where $T$ is a $p$-adic torus and where $\pi$ is a finite $p$-group (as a $p$-compact group ), i.e. $B \pi \simeq K(\pi, 1)$ and $\pi$ is an honest finite group. For every $p$-compact toral group $P$, there exists a locally finite group $P_{\infty}$, which actually is the union of finite groups, and a map $B P_{\infty} \rightarrow B P$ which becomes an equivalence after completion. $P_{\infty} \rightarrow P$ is called the $p$-discrete approximation of $P[5,6.4]$.

2.3 Conjugation and subconjugation. Two homomorphisms $f, g: Y \rightarrow X$ of $p$-compact groups are called conjugate if the induced maps $B f, B g: B Y \rightarrow B X$ are freely homotopic.

For a homomorphism $f: Y \rightarrow X$ of $p$-compact groups and for a $p$-compact toral subgroup $i: P \rightarrow X$ we say that $P$ is subconjugate to $Y$ if there exists a homomorphism $j: P \rightarrow Y$ such that $f j$ and $i$ are conjugate.

2.4 Centralizers. For a homomorphism $f: Y \rightarrow X$ between $p$-compact groups, the centralizer $C_{X}(f(Y))$ is defined to be the loop space given by the triple

$$
C_{X}(f(Y)):=\left(\Omega \operatorname{map}(B Y, B X)_{B f}, \operatorname{map}(B Y, B X)_{B f}, i d\right) .
$$

The evaluation at the basepoint $e v: \operatorname{map}(B Y, B X)_{B f} \rightarrow B X$ establishes a homomorphism $C_{X}(f(Y)) \rightarrow X$ of loop spaces. If $Y$ is a $p$-compact toral group, then the centralizer $C_{X}(f(Y))$ is again a $p$-compact group and the evaluation $C_{X}(f(Y)) \rightarrow X$ is a monomorphism [5, 5.1, 5.2 and 6.1].

2.5 Maximal tori. The maximal torus of a $p$-compact group $X$ is a monomorphism $T_{X} \rightarrow X$ of a $p$-compact torus into $X$ such that the centralizer $C_{X}\left(T_{X}\right)$ is a $p$-compact toral group whose component of the unit is given by $T_{X}$ [5]. Because $T_{X}$ centralizes itself, there is a homomorphism $T_{X} \rightarrow C_{X}\left(T_{X}\right)$

2.6 Theorem [5, 8.11, 8.13 and 9.1]. Let $X$ be a p-compact group.

1. $X$ has a maximal torus $T_{X} \rightarrow X$.

2. Any subtorus $T \rightarrow X$ of $X$ is subconjugated to the maximal torus $T_{X} \rightarrow X$.

3. Any two maximal tori of $X$ are conjugate.

4. If $X$ is connected, then $T_{X} \rightarrow C_{X}\left(T_{X}\right)$ is an isomorphism.

5. If $X$ is connected, then every finite cyclic subgroup $\mathbb{Z} / p^{n} \rightarrow X$ of $X$ is subconjugate to $T_{X}$.

2.7 Proposition. Let $X$ be a p-compact group, and let $T \rightarrow X$ be a monomorphism of a p-compact torus into $X$. Then the following conditions are equivalent:

1. $r k(T)=r k(X)$.

2. The homomorphism $T \rightarrow X$ is a maximal torus. 
Proof. Let $T \rightarrow X$ be a subtorus satisfying condition (1). By Theorem 2.6, there exists a maximal torus $T_{X} \rightarrow X$, and $T \rightarrow X$ lifts up to conjugation to a monomorphism $T \rightarrow T_{X}$. This is an isomorphism because of the rank condition, and $T \rightarrow X$ is a maximal torus.

If $T_{X} \rightarrow X$ is a maximal torus, then $r k(X)=r k(T)$ (Theorem 2.9 (2)), and hence $T \rightarrow X$ satisfies condition (1).

2.8 Weyl spaces and Weyl groups. Let $T_{X} \rightarrow X$ be a maximal torus of a $p$-compact group. We think of $B T_{X} \rightarrow B X$ as being a fibration. The Weyl space $\mathcal{W}_{T}(X)$ is defined to be the mapping space of all fiber maps over the identity on $B X$. Then each component of $\mathcal{W}_{T}(X)$ is contractible and the Weyl group $W_{T}(X):=\pi_{0}\left(\mathcal{W}_{T}(X)\right)$ is a finite group under composition $[5,9.5]$.

2.9 Theorem [5, 9.5 and 9.7]. Let $T_{X} \rightarrow X$ be the maximal torus of a connected p-compact group $X$.

1. The action of $W_{X}$ on $B T_{X}$ induces representations

$$
W_{X} \rightarrow \operatorname{Aut}\left(H^{2}\left(B T_{X} ; \mathbb{Z}_{p}^{\wedge}\right) \otimes \mathbb{Q}\right) \cong G l\left(n, \mathbb{Q}_{p}^{\wedge}\right)
$$

and

$$
W_{X} \rightarrow \operatorname{Aut}\left(H_{2}\left(B T_{X} ; \mathbb{Z}_{p}^{\wedge}\right) \otimes \mathbb{Q}\right) \cong G l\left(n, \mathbb{Q}_{p}^{\wedge}\right)
$$

which are monomorphisms whose images are generated by pseudo reflections.

2. The map $H_{\mathbb{Q}_{p}^{\wedge}}^{*}(B X) \rightarrow H_{\mathbb{Q}_{p}^{\wedge}}^{*}\left(B T_{X}\right)^{W_{X}}$ is an isomorphism.

Here, we define $H_{\mathbb{Q}_{p}^{\wedge}}^{*}(-):=H^{*}\left(-; \mathbb{Z}_{p}^{\wedge}\right) \otimes \mathbb{Q}$.

2.10 Proposition. Let $X$ be a connected $p$-compact group. Then $H^{2}\left(B X ; \mathbb{Z}_{p}^{\wedge}\right) \rightarrow$ $H^{2}\left(B T_{X} ; \mathbb{Z}_{p}^{\wedge}\right)^{W_{X}}$ is an isomorphism.

Proof. The statement is true for simply connected $X$, because $H^{2}\left(B T_{X} ; \mathbb{Z}_{p}^{\wedge}\right)^{W_{X}}$ $\subset H_{\mathbb{Q}_{p}^{\hat{p}}}^{2}\left(B T_{X}\right)^{W_{X}} \cong H_{\mathbb{Q}_{p}^{\hat{p}}}^{2}(B X)=0$ in this case.

For the general case, we recall [13] that there exists a diagram of exact sequences

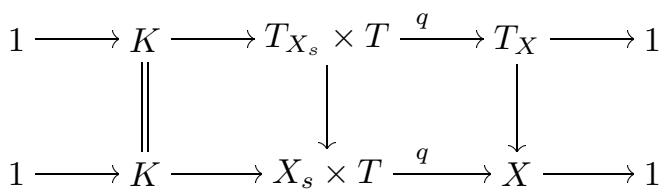

of $p$-compact groups, where $X_{s}$ is simply connected, where $T$ is a $p$-compact torus and where $K \rightarrow X_{s} \times T$ is a central finite subgroup. The projection induces an isomorphism $W_{X_{s}} \cong W_{X}=: W$ between the Weyl groups, which we identify via this map. Then, the upper horizontal line is $W$ equivariant (up to homotopy).

Applying the Serre spectral sequence to both fibrations and passing to invariants establishes a diagram

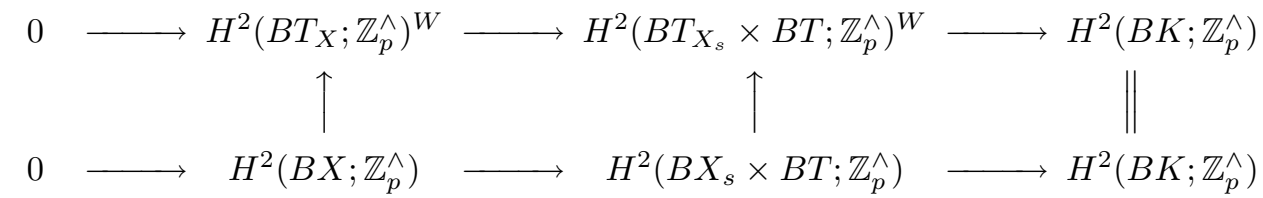

Both rows are exact. The middle terms in both rows are isomorphic to $H^{2}\left(B T ; \mathbb{Z}_{p}^{\wedge}\right)$. For the top row, this follows from Theorem 2.9. Hence, the right and middle vertical 
arrows are isomorphisms, and so is the left one by the five lemma. This finishes the proof.

2.11 Proposition. Let $p$ be an odd prime and $X$ a p-compact group. If the associated representation $W_{X} \rightarrow G l\left(H^{2}\left(B T_{X} ; \mathbb{Z}_{p}^{\wedge}\right)\right.$ is a pseudo reflection group, then $X$ is connected.

Proof. Because $W_{X}$ is a $p$-adic pseudo reflection group, it is generated by elements of order coprime to $p$. By $[13,3.8]$ there exists an epimorphism $W_{X} \rightarrow \pi_{0}(X)$. Because $\pi_{0}(X)$ is a finite $p$-group, this homomorphism is also the trivial map. Thus, $X$ is connected.

2.12 Remark. Let $X$ be a $p$-compact group. The proof of Proposition 2.11 shows that $X$ is connected if any homomorphism $W_{X} \rightarrow \pi$, from the Weyl group into a finite $p$-group, always has a kernel which is not a pseudo reflection group. For example, this is true if $p=2$ and if $X$ has the 2-adic Weyl group type of $G$, where

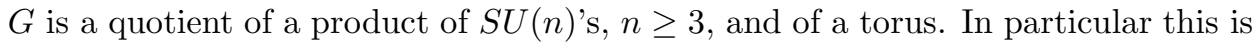
true if $G$ satisfies condition $\left(C_{2}\right)$.

2.13 Normalizers and $p$-normalizers of maximal tori and $p$-toral Sylow subgroups. Let $i: T_{X} \rightarrow X$ be a maximal torus of a $p$-compact group $X$. Again we think of $B T_{X} \rightarrow B X$ as being a fibration. The Weyl space $\mathcal{W}_{X}$ acts on $B T_{X}$ via fiber maps. This establishes a monoid homomorphism $\mathcal{W}_{X} \rightarrow H E\left(B T_{X}\right)$, where $H E\left(B T_{X}\right)$ denotes the monoid of all self-equivalences of $B T_{X}$. Passing to classifying spaces establishes a map $B \mathcal{W}_{X} \rightarrow B H E\left(B T_{X}\right)$, which can be thought of as being a classifying map of a fibration $B T_{X} \rightarrow B N\left(T_{X}\right) \rightarrow B \mathcal{W}_{X}$. The total space gives the classifying space of the normalizer $N\left(T_{X}\right)$ of $T_{X}$. This is always a finite extension of the $p$-compact torus $T_{X}$.

Let $\mathcal{W}_{p}$ be the union of those components of $\mathcal{W}_{X}$ corresponding to a $p$-Sylow subgroup $W_{p}$ of $W_{X}$. The restriction of the above construction to $\mathcal{W}_{p}$ gives the classifying space of the $p$-normalizer $N_{p}\left(T_{X}\right)$.

Since the action of $\mathcal{W}_{X}$ respects the map $B T_{X} \rightarrow B X$, the monomorphism $T_{X} \rightarrow X$ extends to a loop map $N\left(T_{X}\right) \rightarrow X$.

2.14 Proposition. For a p-compact group $X$, the map

$$
H^{*}\left(B X ; \mathbb{F}_{p}\right) \rightarrow H^{*}\left(B N_{p}\left(T_{X}\right) ; \mathbb{F}_{p}\right)
$$

is a monomorphism.

Proof. This follows from [5, proof of 2.3 and 9.13].

2.15 Centers. A subgroup $Z \rightarrow X$ of a $p$-compact group $X$ is called central [5] if the homomorphism $C_{X}(Z) \rightarrow X$ is an isomorphism. The center $Z(X)$ of $X$ is the maximal central subgroup of $X[6,1.2],[13,4.3,4.4]$. To give an explicit definition we use a result of Dwyer and Wilkerson $[6,1.3]$. For every $p$-compact group $X$, the centralizer $C_{X}(X)$ is a $p$-compact group and $Z(X):=C_{X}(X) \rightarrow X$ is the center of $X$.

For every $p$-compact group $X$ there exists a short exact sequence $Z(X) \rightarrow X \rightarrow$ $X / Z(X)=: P X$ of $p$-compact groups, and, if $X$ is connected, the quotient $P X$ has a trivial center $[13,4.7]$. We call a $p$-compact group $X$ centerfree if $Z(X)$ is the trivial group.

For connected $p$-compact groups, there is another description of the center in terms of the fixed point set of the Weyl group action on the maximal torus. Let 
$T_{X} \rightarrow X$ be the maximal torus of a connected $p$-compact group $X$. The Weyl group $W_{X}$ acts on the $p$-discrete approximation $\check{T} \subset T_{X}$ of $T_{X}$. Here, we can consider $\check{T}$ as an honest locally finite group with an honest $W_{X}$-action. Then we define $T_{X}^{W_{X}}$ to be the $p$-compact group given by the equivalence $B T_{X}^{W_{X}} \simeq B\left(\check{T}^{W_{X}}\right)_{p}^{\wedge}$ between the classifying spaces.

2.16 Proposition. Let $X$ be a connected $p$-compact group. If one of the following three conditions is satisfied, then $T_{X}^{W_{X}} \rightarrow X$ is the center of $X$.

1. $p$ is odd.

2. $T_{X}^{W X}$ is connected; i.e. $T_{X}^{W X}$ is a p-compact torus.

3. $H^{*}\left(B X ; \mathbb{F}_{p}\right) \cong H^{*}\left(B T_{X} ; \mathbb{F}_{p}\right)^{W_{X}}$.

Proof. If $p$ is odd, this is proved in [6, 7.7]. If $T_{X}^{W_{X}}$ is a $p$-compact torus, then $C:=C_{X}\left(T_{X}^{W_{X}}\right)$ is connected $[13,3.11]$ with Weyl group $W_{C}=W_{X}$. Therefore, $C \rightarrow X$ induces an isomorphism in rational cohomology between the classifying spaces (Theorem 2.9) and between the spaces itself, and is even an isomorphism between connected $p$-compact groups [13, 3.7].

If $H^{*}\left(B X ; \mathbb{F}_{p}\right) \cong H^{*}\left(B T_{X} ; \mathbb{F}_{p}\right)^{W_{X}}$, then

$$
H^{*}\left(B C_{X}\left(T_{X}^{W_{X}}\right) ; \mathbb{F}_{p}\right) \cong H^{*}\left(B T_{X} ; \mathbb{F}_{p}\right)^{W^{\prime}}
$$

$[15,10.1]$. Here, the subgroup $W^{\prime} \subset W_{X}$ consists of all elements acting trivially on $T_{X}^{W_{X}}$. That is to say that $W^{\prime}=W_{X}$. Therefore, $B C_{X}\left(T_{X}^{W_{X}}\right) \rightarrow B X$ is a homotopy equivalence and $C_{X}\left(T_{X}^{W_{X}}\right) \rightarrow X$ an isomorphism.

\section{The Weyl group of a CONneCted finite loop SPaCe}

In this section $L$ always denotes a connected finite loop space with maximal torus $f: T_{L} \rightarrow L$. We are going to discuss the Weyl group $W_{L}$ of $L$. In particular we will prove Theorem 1.2.

Passing to completion at a prime $p$, we get a $p$-compact group $L_{p}^{\wedge}$ with maximal torus $T_{L p} \hat{p} \rightarrow L_{p}^{\wedge}$ and Weyl group $W_{L_{p}}$. These pieces satisfy the identity $H_{\mathbb{Q}_{p}^{\wedge}}^{*}\left(B L_{p}^{\wedge}\right) \cong H_{\mathbb{Q}_{p}^{\wedge}}^{*}\left(B T_{L p} \wedge\right)^{W_{L_{p}}}$ by Theorem 2.9. That is to say that the monomorphism $H_{\mathbb{Q}_{p}^{\wedge}}^{*}\left(B L_{p}^{\wedge}\right) \rightarrow H_{\mathbb{Q}_{\hat{p}}^{*}}^{*}\left(B T_{L p} \hat{\wedge}\right)$ is a Galois extension of integral domains which are integrally closed with Galois group $W_{L_{\hat{p}}}$. An integral domain is called integrally closed if it is integrally closed in its field of fractions.

To get global information we have to study the rational situation. First we have to clarify and to fix some notions and notations about integral ring extensions. We denote by $F F(A)$ the field of fractions of an integral domain $A$. An integral extension $A \rightarrow B$ of integral domains is called normal, separable or Galois if the associated extension $F F(A) \rightarrow F F(B)$ of the field of fractions is normal, separable or Galois.

3.1 Lemma. Let $A \rightarrow B$ be a Galois extension of integral domains $A$ and $B$, and let $W$ be the Galois group of $F F(A) \rightarrow F F(B)$. If $A$ and $B$ are integrally closed, then $A=B^{W}$.

Proof. Because $A \rightarrow B$ is an integral extension and because $B$ is integrally closed, $B$ is also the integral closure of $A$ in $F F(B)$. Therefore, by naturality, $W$ acts on $B$.

If $b \in B^{W}$, then $b$ is integral over $A$ and $b \in F F(A)$. This implies that $b \in A$, and hence $A=B^{W}$. 
3.2 Proposition. Let $L$ be a connected finite loop space with maximal torus $f$ : $T_{L} \rightarrow L$. The map $H^{*}(B L ; \mathbb{Q}) \rightarrow H^{*}\left(B T_{L}\right)$ is a monomorphism and an integral Galois extension of integrally closed domains.

Proof. Let $A:=H^{*}(B L ; \mathbb{Q})$ and $B:=H^{*}\left(B T_{L} ; \mathbb{Q}\right)$. Then, $A$ and $B$ are polynomial rings on $n=r k(L)=r k\left(T_{L}\right)$ generators. In particular, they are integrally closed domains and noetherian. Because the homogeneous space $L / T_{L}$ is $\mathbb{Z}$-finite, a Serre spectral sequence argument shows that $B$ is a finitely generated $A$-module. Because $A$ and $B$ have the same transcendence degree over $\mathbb{Q}$, the map $A \rightarrow B$ is an inclusion. This shows that $A \rightarrow B$ is an integral ring extension [10, XI, $\S 1$ ].

The characteristic of $\mathbb{Q}$ is zero, and therefore all extensions are separable. So it only remains to show that $A \rightarrow B$ is normal. Tensoring with $\mathbb{Z}_{p}^{\wedge}$ yields $A \otimes \mathbb{Z}_{p}^{\wedge} \cong$ $H_{\mathbb{Q}_{p}^{\wedge}}^{*}\left(B L_{p}^{\wedge}\right)$ and $B \otimes \mathbb{Z}_{p}^{\wedge} \cong H_{\mathbb{Q}_{p}^{\wedge}}^{*}\left(B T_{L p} \hat{)}\right)$. Therefore, by Theorem $2.9, A \otimes \mathbb{Z}_{p}^{\wedge} \cong$ $\left(B \otimes \mathbb{Z}_{p}^{\wedge}\right)^{W_{L_{p}}} \rightarrow B \otimes \mathbb{Z}_{p}^{\wedge}$ is an integral Galois extension of integrally closed domains. Now we can proceed as in $[18,3.5]$ to show that $A \rightarrow B$ is normal and hence a Galois extension.

Proof of Proposition 1.8. Part (1) is obvious, as $\mathbb{Z}$-finiteness implies $\mathbb{F}_{p}$-finiteness and for a simply connected space $Y$ we have $\Omega\left(Y_{p}^{\wedge}\right) \simeq(\Omega Y)_{p}^{\wedge}$ [4, VI, 6.5]. For (2), the same argument shows that $T_{L p} \wedge L_{p}^{\wedge}$ is a maximal torus. The isomorphism between the Weyl groups is shown in the proof of Theorem 1.2 below.

Proof of Theorem 1.2. Let $W_{\mathbb{Q}}$ be the Galois group of the extension $H^{*}(B L ; \mathbb{Q}) \rightarrow$ $H^{*}\left(B T_{L} ; \mathbb{Q}\right)$. The Weyl group $W_{p}^{\wedge}$ of the $p$-compact group $L_{p}^{\wedge}$ is the Galois group of $H^{*}(B L ; \mathbb{Q}) \otimes \mathbb{Z}_{p}^{\wedge} \rightarrow H^{*}\left(B T_{L} ; \mathbb{Q}\right) \otimes \mathbb{Z}_{p}^{\wedge}$ (Theorem 2.9). So, the functor $\otimes \mathbb{Z}_{p}^{\wedge}$ induces a group homomorphism $W_{\mathbb{Q}} \rightarrow W_{p}^{\wedge}$. Because the completion $T_{p}^{\wedge} \rightarrow L_{p}^{\wedge}$ of the maximal torus of $L$ is a maximal torus of $L_{p}^{\wedge}$ (Proposition 1.8), both groups $W_{\mathbb{Q}}$ and $W_{p}^{\wedge}$ are faithfully represented by the action on $H^{*}(B T ; \mathbb{Q}) \otimes \mathbb{Z}_{p}^{\wedge} \cong H^{*}\left(B T_{p}^{\wedge} ; \mathbb{Z}_{p}^{\wedge}\right) \otimes \mathbb{Q}$. Because

$$
\left(H^{*}(B T ; \mathbb{Q}) \otimes \mathbb{Z}_{p}^{\wedge}\right)^{W_{\mathbb{Q}}} \cong H^{*}(B L ; \mathbb{Q}) \otimes \mathbb{Z}_{p}^{\wedge} \cong\left(H^{*}\left(B T_{p}^{\wedge} ; \mathbb{Z}_{p}^{\wedge}\right) \otimes \mathbb{Q}\right)^{W_{p}^{\wedge}},
$$

the homomorphism $W_{\mathbb{Q}} \rightarrow W_{p}^{\wedge}$ is an isomorphism.

In the diagram

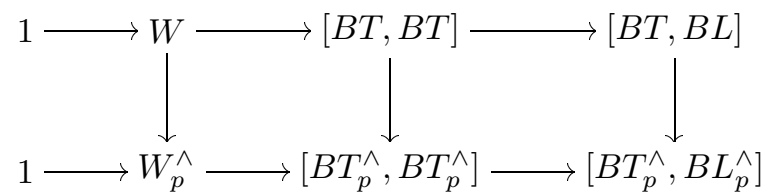

both rows are exact. For the top row this follows from the definition, and for the bottom row from Theorem 2.9. Hence, the third term in the rows contains the quotient of the first two terms as a subset. Here, $W$ denotes the Weyl monoid of $L$. Therefore, $p$-completion induces for every prime $p$ a monoid homomorphism $W \rightarrow W_{p}^{\wedge}$ which is injective. This map is also surjective. For let $w_{p} \in W_{p}^{\wedge}$ for some prime $p$. We choose elements $w_{0} \in W_{\mathbb{Q}}$ and $w_{q} \in W_{q}^{\wedge}$ corresponding under the isomorphisms $W_{q}^{\wedge} \cong W_{\mathbb{Q}} \cong W_{p}^{\wedge}$. We realize $w_{0}$ as a self map of $B T_{\mathbb{Q}}$ over $B L_{\mathbb{Q}}$, and let $w_{q}$ denote the associated self map of $B T_{q}^{\wedge}$ over $B L_{q}^{\wedge}$. Since $H^{2}\left(B T_{p}^{\wedge} ; \mathbb{Z}_{p}^{\wedge}\right) \otimes \mathbb{Q} \cong$ $H^{2}\left(B T_{\mathbb{Q}} ; \mathbb{Q}\right) \otimes \mathbb{Z}_{p}^{\wedge}$ for all primes, an arithmetic square argument shows that there exists a self map $w: B T \rightarrow B T$ over $B L$ such that $w_{q}^{\wedge} \simeq w_{q} \in\left[B T_{q}^{\wedge}, B T_{q}^{\wedge}\right]$. In particular, we have $W \cong W_{p}^{\wedge}$, and $W$ is a group, which is part (1). 
Because $W$ is a subgroup of $[B T, B T]$, the integral cohomology group $H^{2}(B T ; \mathbb{Z})$ gives an invariant sublattice of $H^{2}(B T ; \mathbb{Q})$. Moreover, we have $H^{*}(B T ; \mathbb{Q})^{W} \cong$ $H^{*}(B T ; \mathbb{Q})^{W_{\mathbb{Q}}} \cong H^{*}(B L ; \mathbb{Q})$. Because $H^{*}(B L ; \mathbb{Q})$ is a polynomial ring, the action of $W$ on $H^{2}(B T ; \mathbb{Q})$ represents $W$ as a reflection group. This proves the last two parts of the statement.

The proof of Theorem 1.2 shows that the integral Weyl group $W_{L}$ and the $p$ adic Weyl groups $W_{L \wedge}$ of a connected finite loop space $L$ with maximal torus $T_{L} \rightarrow L$ are isomorphic for every prime $p$. We identify all these Weyl groups via this isomorphism. Using this fact one also can construct an integral normalizer of the maximal torus $T_{L} \rightarrow L$, which is the claim of Proposition 1.4.

Fibrations of the form $B T_{L} \rightarrow Y \rightarrow B W_{L}$ are classified by cohomology classes in $H^{3}\left(B W_{L} ; L T_{L}\right)$, where $W_{L}$ acts on $L T_{L}=H_{2}\left(B T_{L} ; \mathbb{Z}\right)$ via the action on the maximal torus. Fibrations over $B W$ with fiber $B T_{L p} \wedge$ are classified by cohomology classes in $H^{3}\left(B W_{L} ; L T_{L p} \wedge\right)$. In both cases this follows from obstruction theory. Because $W_{L}$ is a finite group we have an isomorphism $H^{3}\left(B W_{L} ; L T_{L}\right) \cong$ $\prod_{p} H^{3}\left(B W_{L} ; L T_{L p} \wedge\right)$. This isomorphism is given by the product over all $p$-adic fiberwise completions. Hence there exists a fibration $B T_{L} \rightarrow B N \rightarrow B W$, such that fiberwise completion induces an equivalence $B N_{p}^{\circ} \simeq B N\left(T_{L_{p}}\right)$. Moreover, by $[16,3.2]$, the space $B N$ is the classifying space of a uniquely determined compact Lie group $N$ fitting into a short exact sequence $1 \rightarrow T_{L} \rightarrow N \rightarrow W \rightarrow 1$. In particular, the group $N$ gives a finite loop space, which we call the normalizer of the maximal torus $T_{L} \rightarrow L$ of $L$.

Next we want to construct a map $B N \rightarrow B L$. By the construction of $N$, for every prime $p$, there exists a map $B N \rightarrow B L_{p}^{\wedge}$ which is an extension of the map $B T_{L} \rightarrow B L_{p}^{\wedge}$ and which induces an isomorphism $H_{\mathbb{Q}_{p}^{\wedge}}^{*}(B N) \cong H_{\mathbb{Q}_{p}^{\wedge}}^{*}(B L)$. The isomorphism between the cohomology groups follows from the Serre spectral sequence for calculating the cohomology of $B N$ and Theorem 2.9. The same argument using Theorem 1.2 instead of Theorem 2.9 shows that there exists an isomorphism $H^{*}(B N ; \mathbb{Q}) \cong H^{*}(B L ; \mathbb{Q})$. Because $B L_{\mathbb{Q}}$ is a product of Eilenberg-Mac Lane spaces, this last isomorphism can be realized by a map $B N \rightarrow B L_{\mathbb{Q}}$. All coherence conditions for using an arithmetic square are controlled by cohomology and are therefore satisfied by construction. This establishes a map $B N \rightarrow B L$ which, again by construction, is an extension of $B T_{L} \rightarrow B L$. We collect the properties of the group $N$, now called $N\left(T_{L}\right)$, in the following statement, which also includes Proposition 1.4.

3.4 Proposition. Let $L$ be a connected finite loop space with maximal torus $T_{L} \rightarrow$ $L$ and Weyl group $W_{L}$. Then the following hold:

1. The triple $\left(N\left(T_{L}\right), B N\left(T_{L}\right), e\right)$ is a finite loop space. In particular, $N\left(T_{L}\right)$ is isomorphic to a uniquely determined compact Lie group given as a finite extension of $T_{L}$ by $W_{L}$.

2. There exists a homomorphism $N\left(T_{L}\right) \rightarrow L$ of finite loop spaces which is an extension of $T_{L} \rightarrow L$.

Finally we show, as promised in the introduction, that any two maximal tori of a finite loop space are conjugate.

3.5 Proposition. Let $T \stackrel{g}{\rightarrow} L$ be a homomorphism of a torus into a connected finite loop space $L$ with maximal torus $T_{L} \stackrel{f}{\rightarrow} L$. Then $T$ is subconjugate to $T_{L} \stackrel{f}{\rightarrow} L$. 
Proof. Let $A:=H^{*}(B L ; \mathbb{Q}), B:=H^{*}\left(B T_{L} ; \mathbb{Q}\right)$ and $C:=H^{*}(B T ; \mathbb{Q})$, and let $A_{p}^{\wedge}, B_{p}^{\wedge}$ and $C_{p}^{\wedge}$ denote the associated cohomology groups given by the theory $H_{\mathbb{Q}_{\hat{p}}}^{*}()$. After completion at a prime $p$, we already know that $T$ is subconjugate to $T_{L \hat{p}} \rightarrow L_{p}^{\wedge}$ via a homomorphism $T_{p}^{\wedge} \stackrel{h_{p}^{\wedge}}{\longrightarrow} T_{L p} \wedge$. Therefore, the diagram

$$
\begin{array}{rl}
B f^{*} & \stackrel{B h^{*}}{\longrightarrow} C_{p}^{\wedge} \\
A & \left.B g^{*}\right|_{p} ^{\wedge}
\end{array}
$$

commutes. Let $p(t) \in A[t]$ be a monic polynomial which splits into linear factors over $B$. By the diagram $(*)$, the polynomial $B g^{*}(p(t)) \in C[t]$ also splits into linear factors after completion at every prime. Now we can argue as in the proof of $[18,3.5]$ to show that $B g^{*}(p(t))$ already splits into linear factors over $C$. Because $A \rightarrow B$ is an integral Galois extension, there exists an extension $\phi: B \rightarrow C$ of $B g^{*}: A \rightarrow C$. Because all involved spaces are rationally products of Eilenberg-Mac Lane spaces, the map $\phi$ has a geometric realization $B h_{0}: B T_{0} \rightarrow\left(B T_{L}\right)_{0}$ which is a lift of $B T_{0} \stackrel{B g}{\longrightarrow} B L_{0}$.

Over the adeles, the maps $B h_{0}$ and $B h_{p}^{\wedge}$ differ only by an Weyl group element [2]. Hence we can assume that they are equal over the adeles. In this case, the coherence conditions for glueing all the maps together by an arithmetic square are satisfied. We get a map $B h: B T \rightarrow B T_{L}$, such that the diagram

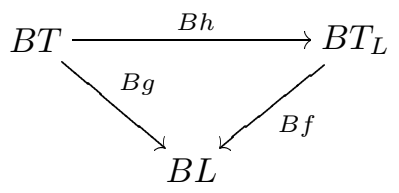

commutes up to homotopy.

The following corollary is obvious.

3.6 Corollary. Let $T_{1}, T_{2} \rightarrow L$ be two maximal tori of a connected finite loop space. Then $T_{1}$ and $T_{2}$ are conjugate.

In particular, this corollary says that the definition of the Weyl group does not depend in an essential way on the chosen maximal torus.

$$
\text { 4. The map }\left[, B T_{X}\right] \rightarrow[, B X]
$$

In this section, $X$ is a $p$-compact group with a fixed maximal torus $f: T_{X} \rightarrow X$. For any $p$-compact toral group $P$, the Weyl group $W_{X}$ acts on the set $\left[B P, B T_{X}\right]$ of homotopy classes of maps and establishes a map

$$
F:\left[B P, B T_{X}\right] / W_{X} \rightarrow[B P, B X]
$$

4.1 Proposition. Let $P$ be a p-compact toral group. The map

$$
F:\left[B P, B T_{X}\right] / W_{X} \rightarrow[B P, B X]
$$

is an injection.

Remark. In $[12,2.3]$ a similar but slightly weaker result is proved with different methods. 
For the proof of this result we will use the following construction. Let $A$ be a $p$-compact abelian group and $\alpha: A \rightarrow T_{X}$ a homomorphism. Both classifying spaces $B A$ and $B T_{X}$ are loop spaces and carry a multiplication $\mu$. Because $\alpha$ is a homomorphism, this establish a commutative diagram

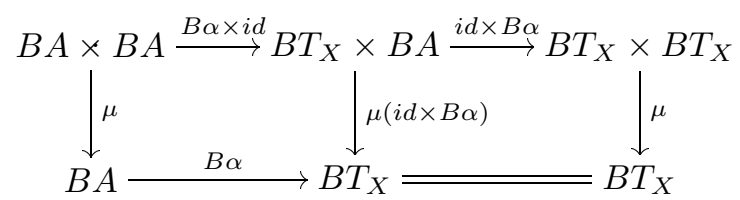

Taking adjoints in the vertical line yields a diagram

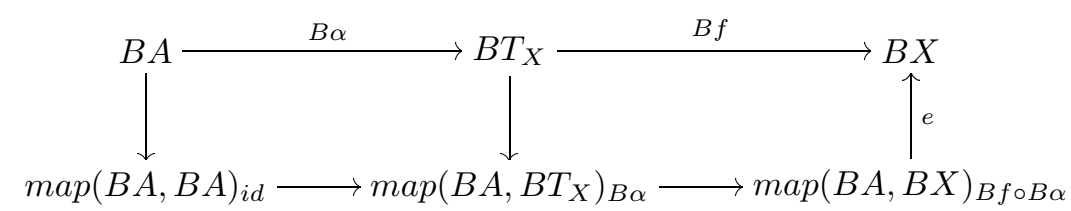

The right vertical arrow is given by the evaluation. Now let $w \in W_{X}$ be an element of the Weyl group. The diagram

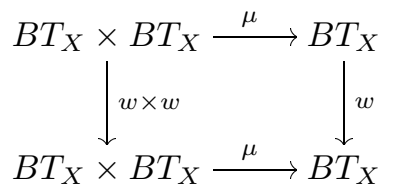

commutes up to homotopy and, taking adjoints again and combining it with $(*)$, establishes another homotopy commutative diagram, namely

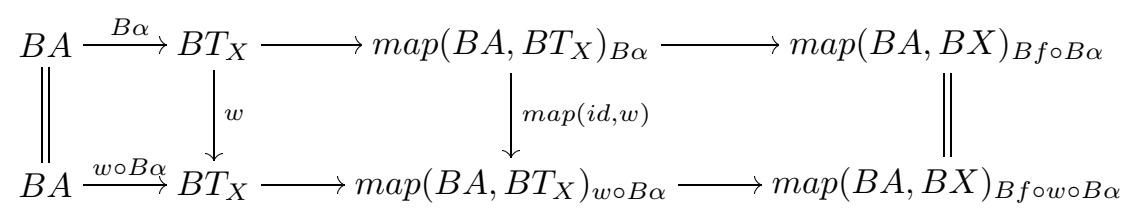

The component of $\operatorname{map}\left(B A, B T_{X}\right)$ in the bottom row is determined by the homotopy commutative square

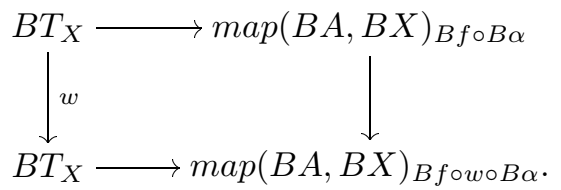

Now we are prepared for the proof of Proposition 4.1.

Proof of Proposition 4.1. Let $\alpha, \beta: P \rightarrow T_{X}$ be two homomorphism such that $f \circ \alpha$ and $f \circ \beta$ are conjugate. First of all this implies that $\alpha$ and $\beta$ have the same kernel which we can divide out. This follows from [17, §2]. There only the case of maps with source given by the classifying space of a honest $p$-toral group is discussed, but the same arguments are applicable in our situation. Hence, we can assume that $\alpha$ and $\beta$ are monomorphisms and that $P$ is abelian as a subgroup of $T_{X}[13,3.1$ 
and 3.5]. We get the following diagram:

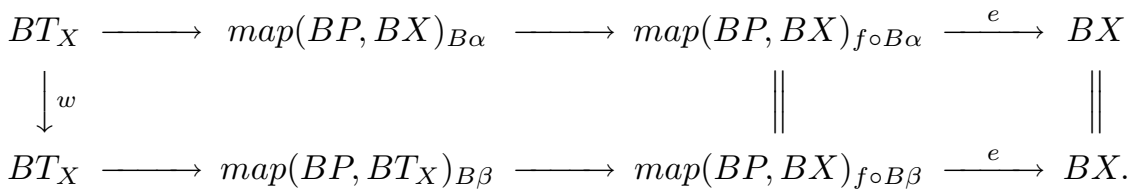

The top and the bottom row give two maximal tori of $\operatorname{map}(B P, B X)_{f \circ B \alpha}$. Hence the left vertical arrow exists and is given by a self equivalence. Because the outer square commutes, this self equivalence is given by an element $w \in W_{X}$. The above considerations imply that $w \circ B \alpha \simeq B \beta$, which finishes the proof.

Next we will discuss under which conditions the map is also a surjection. We have the following generalization of well known results of Borel.

4.2 Theorem. Let $X$ be a connected p-compact group with maximal torus $f$ : $T_{X} \rightarrow X$. Then the following hold:

1. The map $H^{*}\left(B X ; \mathbb{Z}_{p}^{\wedge}\right) \stackrel{B f^{*}}{\longrightarrow} H^{*}\left(B T_{X} ; \mathbb{Z}_{p}^{\wedge}\right)^{W_{X}}$ is an isomorphism if and only if $H^{*}\left(B X ; \mathbb{Z}_{p}^{\wedge}\right)$ is p-torsion free.

2. If $H^{*}\left(B X ; \mathbb{Z}_{p}^{\wedge}\right)$ is $p$-torsion free, every elementary abelian subgroup $V \rightarrow X$ is subconjugate to $T_{X}$.

3. Let $A$ be an abelian p-compact group. If $H^{*}\left(B X ; \mathbb{F}_{p}\right) \cong H^{*}\left(B T_{X} ; \mathbb{F}_{p}\right)^{W_{X}}$, then the map $F:\left[B A, B T_{X}\right] / W_{X} \rightarrow[B A, B X]$ is a bijection.

Proof. First let us assume that $H^{*}\left(X ; \mathbb{Z}_{p}^{\wedge}\right)$ is not torsion free and that $H^{*}\left(B X, \mathbb{Z}_{p}^{\wedge}\right)$ is torsion free. Let $x \in H^{*}\left(X ; \mathbb{Z}_{p}^{\wedge}\right)$ be a torsion class of minimal degree. Then, in the Serre spectral sequence of the fibration $X \rightarrow * \rightarrow B X$ this class can't be killed by any differential, which leads to a contradiction. Hence, if $H^{*}\left(B X ; \mathbb{Z}_{p}^{\wedge}\right)$ is $p$-torsion free then $H^{*}\left(X ; \mathbb{Z}_{p}^{\wedge}\right)$ is also $p$-torsion free. Moreover, $H^{*}\left(X ; \mathbb{F}_{p}\right) \cong E\left(\bar{y}_{1}, \ldots, \bar{y}_{r}\right)$ is an exterior algebra, $H^{*}\left(B X ; \mathbb{F}_{p}\right) \cong \mathbb{F}_{p}\left[\bar{x}_{1}, \ldots, \bar{x}_{r}\right]$ and $H^{*}\left(B X ; \mathbb{Z}_{p}^{\wedge}\right) \cong \mathbb{Z}_{p}^{\wedge}\left[x_{1}, \ldots, x_{r}\right]$ are polynomial algebras, $r$ is equal to the rank $r k_{\mathbb{Q}}(X)$ of $X$ and $H^{*}\left(B X ; \mathbb{Z}_{p}^{\wedge}\right) \stackrel{B f^{*}}{\longrightarrow}$ $H^{*}\left(B T_{X} ; \mathbb{Z}_{p}^{\wedge}\right)^{W_{X}}$ is a monomorphism. All these facts follow from results of Hopf and Borel (e.g. see [9]).

Let $y \in H^{*}\left(B X ; \mathbb{Z}_{p}^{\wedge}\right)$. If $B f^{*}(y) \equiv 0 \bmod p$ then $y \equiv 0 \bmod p$. Otherwise we would get an algebraic relation among the generators of the image of $H^{*}\left(B X ; \mathbb{F}_{p}\right) \rightarrow$ $H^{*}\left(B T_{X} ; \mathbb{F}_{p}\right)$, which contradicts the fact that $H^{*}\left(B T_{X} ; \mathbb{F}_{p}\right)$ is a finitely generated module over $H^{*}\left(B X ; \mathbb{F}_{p}\right)$. On the other hand, for every $x \in H^{*}\left(B T_{X} ; \mathbb{Z}_{p}^{\wedge}\right)^{W_{X}}$, there exist $n \in \mathbb{N}$ and $z \in H^{*}\left(B X ; \mathbb{Z}_{p}^{\wedge}\right)$ such that $B f^{*}(z)=p^{n} x$. This follows from Theorem 2.9. If $n>0$, then $z$ is divisible by $p$ by the above considerations, and $B f^{*}\left(p^{-1} z\right)=p^{-1} x$. This proves (1).

By (1) the map $H^{*}\left(B X ; \mathbb{F}_{p}\right) \rightarrow H^{*}\left(B T_{X} ; \mathbb{F}_{p}\right)$ is an injection. For every elementary abelian $p$-group $V$, the algebra $H^{*}\left(B V ; \mathbb{F}_{p}\right)$ is an injective object in the category of unstable algebras over the Steenrod algebra. Thus, every map $H^{*}\left(B X ; \mathbb{F}_{p}\right) \rightarrow H^{*}\left(B V ; \mathbb{F}_{p}\right)$ lifts to a map $H^{*}\left(B T_{X} ; \mathbb{F}_{p}\right) \rightarrow H^{*}\left(B V ; \mathbb{F}_{p}\right)$. Because in this situation the mod- $p$ cohomology classifies the maps up to homotopy and because every algebraic map has a realization [11], this proves part (2).

The third part is proved in $[15,10.1]$. 


\section{5. $p$-COMPACT GROUPS WITH THE WEYL GROUP TYPE OF UNITARY GROUPS}

If $X$ is a connected $p$-compact group with the same $p$-adic Weyl group type as a compact connected Lie group $G$, then both have the same rank, and we can identify the two maximal tori as well as the Weyl groups. That is to say, there exists a homomorphism $T_{G} \rightarrow X$ which is a maximal torus with Weyl group $W_{G}$. Here, we have to complete $T_{G}$ in order to get a $p$-compact torus. As already said in Section 4, the Weyl group $W_{G}$ acts on the set $\left[B Y, B T_{G}\right]$ of homotopy classes of maps for any $p$-compact group $Y$.

5.1 Theorem. Let $G$ be a product of unitary groups, and let $X$ be a connected $p$-compact group with the same $p$-adic Weyl group type as $G$. Then for any abelian p-compact group $A$ the following hold:

1. $\left[B A, B T_{G p}^{\wedge}\right] \rightarrow[B A, B X]$ is surjective.

2. $\left[B A, B T_{G p} \wedge\right] / W_{G} \rightarrow[B A, B X]$ is a bijection.

3. For any homomorphism $g: A \rightarrow T_{G}$, the centralizer $C_{X}(g)$ is connected and has the p-adic Weyl group type of $C_{G}(g)$, which is a product of unitary groups.

Proof. By Proposition 4.1, the first statement implies the second one.

To prove (1) and (3) we first assume that $A$ is a finite abelian $p$-compact group, i.e $A$ is really a finite abelian $p$-group. Hence, $A$ is isomorphic to a product $A \cong$ $A_{1} \times \cdots \times A_{r}$ such that $A_{i} \cong \mathbb{Z} / p^{k_{i}}$. Moreover, every map $B A \rightarrow B T_{G p} \wedge$ lifts to a map $B A \rightarrow B T_{G}$, and any map $B A \rightarrow B G_{p}^{\wedge}$ lifts to $B G$. By [7] in both cases the lifts are induced by a homomorphism $A \rightarrow T_{G}$ or $A \rightarrow G$ of groups. For the proof of (1) and (3) we will use an induction over the number of factors of $A$.

Let $A \cong \mathbb{Z} / p^{k}$. Then the map $g: A \rightarrow X$ factors through the maximal torus, because every finite cyclic group is subconjugated to the maximal torus (Theorem 2.6). This proves (1) in the case of a cyclic group.

Because $A$ is a subgroup of $T_{G}$, the centralizer $C_{X}(A)$ contains $T_{G}$, which also plays the role of the maximal torus of $C_{X}(A)$. Let $W_{C}$ denote the Weyl group of $C_{X}(A)$, which is isomorphic to the isotropy group $I s o(g):=\left\{w \in W_{G} \mid w \circ B g \simeq\right.$ $B g\}$. By the assumptions this is also the Weyl group of $C_{G}(A)$, which is again a product of unitary groups. Therefore, $T_{G}^{W_{C}}$ is a $p$-compact torus and contains $A$. Moreover, $T_{G}^{W_{C}} \rightarrow C_{X}(A)$ is the center (Proposition $2.16(2)$ ), while $C_{X}(A) \cong$ $C_{C_{X}(A)}\left(T_{G}^{W_{C}}\right) \cong C_{X}\left(T_{G}^{W_{C}}\right)$ is a connected $p$-compact group [13, 3.11] and has the $p$-adic Weyl group type of $C_{G}(A)$, which is a product of unitary groups. This is condition (3).

Now let $A$ be an abelian finite $p$-group. Then $A$ splits into a product $A_{0} \times A_{1}$ such that $A_{1} \cong \mathbb{Z} / p^{k}$ and such that $A_{0}$ has less factors than $A$. Let $g: A \rightarrow X$ be a homomorphism. By the induction hypothesis the restriction $\left.g\right|_{A_{0}}: A_{0} \rightarrow X$ is subconjugate to the maximal torus, and $C_{X}\left(g\left(A_{0}\right)\right)$ is connected and again of the $p$-adic Weyl group type of a product of unitary groups. Passing to adjoints yields a homomorphism $\bar{g}: A_{1} \rightarrow C_{X}\left(g\left(A_{0}\right)\right)$. We can again apply the induction hypothesis to show that $A_{1}$ is subconjugate to $T_{G}$ in $C_{X}\left(g\left(A_{0}\right)\right)$ which proves the first statement, and also shows that $C_{X}(g(A))=C_{C_{X}\left(g\left(A_{0}\right)\right)}\left(\bar{g}\left(A_{1}\right)\right)$ is connected and of the $p$-adic Weyl group type of a product of unitary groups, which is part (3).

Finally let $A$ be an abelian $p$-compact toral group. Let $A(k) \rightarrow A$ be the subgroup of elements of order $p^{k}$. Then $A(\infty):=\bigcup_{k} A(k) \rightarrow A$ is a $p$-discrete 
approximation. The map $\operatorname{map}(B A, B X) \rightarrow \varliminf_{k} \operatorname{map}(B A(k), B X)$ is an equivalence. Moreover, for every fixed homomorphism $f: A \rightarrow X$, the sequence $\operatorname{map}(B A(k), B X)_{\left.B f\right|_{B A(k)}}$ stabilizes [5,6.1,6.7]. This proves the statement in the general case.

For odd primes there is a more general result of this form.

5.2 Theorem. Let $p$ be an odd prime, $G$ a compact connected Lie group satisfying condition $\left(T_{p}\right)$, and $X$ a connected $p$-compact group with the same $p$-adic Weyl group type as $G$. Then the following hold:

1. $\left[B A, B T_{G p}{ }^{\wedge}\right] \rightarrow[B A, B X]$ is an epimorphism.

2. $\left[B A, B T_{G p} \wedge\right] / W_{G} \rightarrow[B A, B X]$ is an isomorphism.

3. For any homomorphism $g: A \rightarrow T_{G}$, the centralizer $C_{X}(g)$ is connected and has the p-adic Weyl group type of $C_{G}(g)$. Moreover, $C_{G}(g)$ satisfies condition $\left(T_{p}\right)$.

Proof. We argue as in the above proof. Only one argument has to be replaced. We use the same notation as above. In this case $T_{G}^{W_{C}}$ may not be a torus. But by $[15$, 10.1] we have

$$
H^{*}\left(B C_{G}(g(A)) ; \mathbb{F}_{p}\right) \cong H^{*}\left(B T_{G} ; \mathbb{F}_{p}\right)^{I s o(g)},
$$

where $I s o(g):=\left\{w \in W_{G} \mid w \circ B g \simeq B g\right\}$ is the isotropy group of $g$. In particular, $C_{G}(g(A))$ is connected with Weyl group $W_{C} \cong I s o(g)$, the mod- $p$ cohomology is concentrated in even degrees, and $C_{X}(g(A))$ is a $p$-compact group with the same Weyl group type as $C_{G}(g(A))$. Because $W_{C}$ is a reflection group and because $p$ is odd, $C_{X}(g(A))$ is connected (Lemma 2.11). Using this argument, the induction of the proof of Theorem 5.1 works and gives a proof of the statement.

One part of Theorem 1.9 for products of unitary groups is contained in the following statement:

Theorem 5.3. Let $X$ be a connected $p$-compact group with maximal torus, and let $G$ be a product of unitary groups. If $B N\left(T_{X}\right)$ and $B N\left(T_{G}\right)_{p}^{\circ}$ are homotopy equivalent, then the following hold:

1. $B X$ is $p$-torsion free, i.e. the $p$-adic cohomology is $p$-torsion free.

2. $H^{*}\left(B X ; \mathbb{Z}_{p}^{\wedge}\right) \cong H^{*}\left(B T_{X} ; \mathbb{Z}_{p}^{\wedge}\right)^{W_{X}}$.

3. $B X$ and $B G_{p}^{\wedge}$ are homotopy equivalent.

Proof. Because the two normalizers $B N\left(T_{X}\right)_{p}^{\circ}$ and $B N\left(T_{G}\right)_{p}^{\circ}$ are equivalent, the space $X$ has the same $p$-adic Weyl group type as $G$. The mod- $p$ cohomology of $B N\left(T_{G}\right)$ is detected by elementary abelian $p$-subgroups [8]. The map $H^{*}\left(B X ; \mathbb{F}_{p}\right)$ $\rightarrow H^{*}\left(B N\left(T_{X}\right) ; \mathbb{F}_{p}\right)$ is a monomorphism (Proposition 2.14). Thus, the cohomology $H^{*}\left(B X ; \mathbb{F}_{p}\right)$ is also detected by elementary abelian $p$-subgroups. By Theorem 5.1, we know that every elementary abelian $p$-subgroup is subconjugate to the maximal torus. This implies that $H^{*}\left(B X ; \mathbb{F}_{p}\right)$ is concentrated in even degrees and that $H^{*}\left(B X ; \mathbb{Z}_{p}^{\wedge}\right)$ is $p$-torsion free. By Theorem 4.2 , it follows that

$$
H^{*}\left(B X ; \mathbb{Z}_{p}^{\wedge}\right) \cong H^{*}\left(B T_{G} ; \mathbb{Z}_{p}^{\wedge}\right)^{W_{G}}
$$

Because $H^{*}\left(B T_{G} ; \mathbb{Z}_{p}^{\wedge}\right)^{W_{G}} \otimes \mathbb{F}_{p} \cong H^{*}\left(B T_{G} ; \mathbb{F}_{p}\right)^{W_{G}}$ we get isomorphisms

$$
H^{*}\left(B X, \mathbb{F}_{p}\right) \cong H^{*}\left(B T_{G} ; \mathbb{F}_{p}\right)^{W_{G}} \cong H^{*}\left(B G ; \mathbb{F}_{p}\right) .
$$


Because $G$ is a product of unitary groups we can apply $[15,1.3]$ to conclude that $B X$ and $B G_{p}^{\wedge}$ are homotopy equivalent. That is what we need for all three statements.

For later purposes we need for odd primes a slightly more general result.

5.4 Theorem. Let $p$ be an odd prime and $G$ a product of unitary groups and $S U(p)$ 's. Let $X$ be a connected $p$-compact group with the same $p$-adic Weyl group type as $G$. If $B N\left(T_{X}\right)$ and $B N\left(T_{G}\right)_{p}^{\circ}$ are homotopy equivalent, then the following hold:

1. $B X$ is $p$-torsion free, i.e. the $p$-adic cohomology is $p$-torsion free.

2. $H^{*}\left(B X ; \mathbb{Z}_{p}^{\wedge}\right) \cong H^{*}\left(B T_{X} ; \mathbb{Z}_{p}^{\wedge}\right)^{W_{X}}$.

3. $B X$ and $B G_{p}^{\wedge}$ are homotopy equivalent.

Proof. The proof is the same as above. We only have to ensure that the $\bmod -p$ cohomology of $B N\left(T_{G}\right)$ is detected by elementary abelian subgroups. But this is also true for $S U(p)[15,12.6]$.

5.5 Proposition. Let $G$ be a product of unitary groups, or, if $p$ is odd, let $G$ be a compact connected Lie group satisfying condition $\left(T_{p}\right)$. If $X$ is a connected $p-$ compact group having the same $p$-adic Weyl group type as $G$, then $B N\left(T_{X}\right)$ and $B\left(N T_{G}\right)_{p}^{\circ}$ are homotopy equivalent.

Proof. For the proof we have to discuss extensions, i.e. fibrations, of the form

$$
B T_{G p}^{\wedge} \rightarrow B N_{p}^{\circ} \rightarrow B W_{G}
$$

Fibrations of this form are classified by cohomology classes in $H^{3}\left(B W_{G} ; \pi_{2}\left(B T_{G_{p}}^{\wedge}\right)\right)$ $[16,3.1]$. If $p$ is odd and if $G$ satisfies the condition $\left(T_{p}\right)$, the obstruction group vanishes $[15,5.3]$, and hence there is only one fibration up to fiber homotopy equivalence. Hence, we have $B N\left(T_{X}\right) \simeq B\left(N T_{G}\right)_{p}^{\circ}$.

Now let $G$ be a product of unitary groups. Difficulties arise only for $p=2$, because in this case there is more than one extension in general. By Proposition 2.10, the map $H^{2}\left(B N\left(T_{X}\right) ; \mathbb{F}_{p}\right) \rightarrow H^{2}\left(B T_{X} ; \mathbb{F}_{p}\right)^{W_{G}}$ is an epimorphism. We can apply $[15,11.5]$, which implies that $B N\left(T_{X}\right)$ is equivalent to the fiberwise completion of the classifying space of the semidirect product $T_{G} \rtimes W_{G} \cong N\left(T_{G}\right)$. This proves the statement for products of unitary groups.

Finally we are prepared to prove Theorem 1.9 for products of unitary groups:

Proof of Theorem 1.9 for products of unitary groups. Condition (1) implies (2) by Proposition 5.5, (2) implies (3) by Theorem 5.2, and (3) implies (1) because $X$ and $G$ are isomorphic $p$-compact groups.

\section{Connected $p$-Compact group With the Weyl group type of PSEudo SIMPLY CONNECTED OR SIMPLY CONNECTED COMPACT LIE GROUPS.}

A pseudo simply connected compact Lie group is a product of unitary groups and simple simply connected compact Lie groups not isomorphic to any $S U(n)$. That is why we replace the special unitary factors in a simply connected compact Lie group by the associated unitary groups. 
6.1 Proposition. Let $G$ be a pseudo simply connected compact Lie group satisfying the condition $\left(C_{p}\right)$. Then there exist an elementary abelian subgroup $V \rightarrow T_{G}$, a compact connected Lie group $H$ which is a product of unitary groups and $S U(p)$ 's, and an exact sequence

$$
1 \rightarrow K \rightarrow H \rightarrow C_{G}(V) \rightarrow 1,
$$

where $K$ is a finite central subgroup of $H$ of order coprime to $p$. The centralizer $C_{G}(V)$ is of maximal rank and the index of $\left|W_{G}: W_{C_{G}(V)}\right|$ is also coprime to $p$. The two groups $H$ and $C_{G}(V)$ have the same Weyl group type, $B N\left(T_{H}\right)_{p}^{\circ} \simeq$ $B N\left(T_{C_{G}(V)}\right)_{p}^{\circ}$, and the mod-p cohomology of $B N\left(T_{G}\right)$ is detected by elementary abelian p-groups.

Proof. This statement is Lemma 5.2 of [15]. Only the last three assertions need a remark. They follow from what we have already said and from the fact that the mod- $p$ cohomolgy of $B N\left(T_{H}\right)$ is detected by elementary abelian subgroups [8], [15, $12.6]$.

6.2 Theorem. Let $G$ be a pseudo simply connected compact Lie group satisfying condition $\left(C_{p}\right)$. Let $X$ be a connected p-compact group with the same Weyl group type as $G$. Then the following hold:

1. $H^{*}\left(B X ; \mathbb{Z}_{p}^{\wedge}\right) \cong H^{*}\left(B T_{G} ; \mathbb{Z}_{p}^{\wedge}\right)^{W_{G}}$.

2. $B N\left(T_{G}\right)_{p}^{\circ}$ and $B N\left(T_{X}\right)$ are homotopy equivalent.

3. $B X$ and $B G_{p}^{\wedge}$ are homotopy equivalent.

Proof. For $p=2$, a pseudo simply connected compact Lie group is a product of unitary groups. This case we already discussed in the last section. Therefore we assume that $p$ is odd. Part (2) follows from Proposition 5.5. The $\bmod -p$ cohomology of $B N\left(T_{X}\right) \simeq B N\left(T_{G}\right)_{p}^{\circ}$ is detected by elementary abelian subgroups. This follows from Proposition 6.1. Now, using Theorem 5.2 instead of Theorem 5.1 , we can argue analogously as in Theorem 5.3 or Theorem 5.4 to show that $H^{*}\left(B X ; \mathbb{Z}_{p}^{\wedge}\right) \cong H^{*}\left(B T_{G} ; \mathbb{Z}_{p}^{\wedge}\right)^{W_{G}}$, and, using again [15, 1.3], that $B X \simeq B G_{p}^{\wedge}$. This is part (3) and also implies part (1).

To conclude this section we discuss the case of simply connected compact Lie groups.

6.3 Theorem. Let $G$ be a simply connected compact Lie group satisfying condition $\left(C_{p}\right)$. Let $X$ be a connected $p$-compact group with the same Weyl group type as $G$. Then the following hold:

1. $H^{*}\left(B X ; \mathbb{Z}_{p}^{\wedge}\right) \cong H^{*}\left(B T_{G} ; \mathbb{Z}_{p}^{\wedge}\right)^{W_{G}}$.

2. $B N\left(T_{G}\right)_{p}^{\circ}$ and $B N\left(T_{X}\right)$ are homotopy equivalent.

3. $B X$ and $B G_{p}^{\wedge}$ are homotopy equivalent.

Proof. We split $G$ into a product $G_{1} \times G_{2}$, where $G_{1}$ is the product of all factors isomorphic to any $S U(n)$ and $G_{2}$ collects the other factors. By the assumptions, $S U(2)$ does not occur for $p=2$. By Proposition 2.16, we have $Z(X) \cong\left(T_{G}^{W_{G}}\right)_{p}^{\wedge}=$ $Z\left(G_{1}\right)_{p}^{\wedge} \times Z\left(G_{2}\right)_{p}^{\wedge}$

For each $n$ there exists an exact sequence $1 \rightarrow Z(S U(n)) \rightarrow S U(n) \times S^{1} \rightarrow$ $U(n) \rightarrow 1$. Let $T$ be a torus with one $S^{1}$ for each factor in $G_{1}$, and let $Z\left(G_{1}\right) \rightarrow$ $T$ be the inclusion established by the above exact sequence. This gives rise to 
commutative diagrams of exact sequences

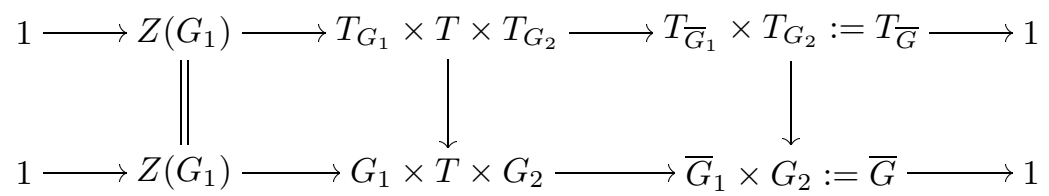

and

$$
1 \rightarrow G \rightarrow \bar{G} \rightarrow \bar{T} \rightarrow 1,
$$

where $Z\left(G_{1}\right) \rightarrow G_{2}$ is the trivial homomorphism, and where $\bar{T}:=T / Z\left(G_{1}\right)$. The quotient $\bar{G}$ is a pseudo simply connected compact Lie group which also satisfies condition $\left(C_{p}\right)$. Moreover, the epimorphism $G \times T \rightarrow \bar{G}$ and the inclusion induce an isomorphism $W_{G} \cong W_{\bar{G}}$, and $T_{G} \times T \rightarrow T_{\bar{G}}$ and $T_{G} \rightarrow T_{\bar{G}}$ are equivariant.

Working with $X$ instead of $G$, one gets similar exact sequences, namely

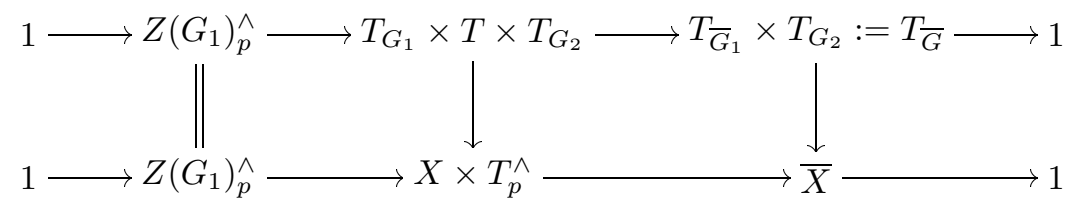

and

$$
1 \rightarrow X \rightarrow \bar{X} \rightarrow \bar{T} \rightarrow 1 \text {. }
$$

This shows that $\bar{X}$ has the same Weyl group type as $\bar{G}$ and hence that $B \bar{X} \simeq B \bar{G}_{p}^{\wedge}$. The maps $B \bar{X} \rightarrow B \bar{T}$ and $B \bar{G} \rightarrow B \bar{T}$ are totally determined by $H^{2}\left(; \mathbb{Z}_{p}^{\wedge}\right)$. And by construction, this establishes a commutative diagram

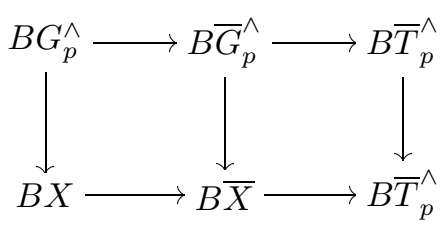

where the right and left vertical arrows are homotopy equivalences. And so is the left one, which is part (3). The first and the second statement obviously follow from this fact.

\section{Connected $p$-COmpact group with the Weyl group type OF A GENERAL COMPACT CONNECTED LIE GROUP}

In this section we want to prove a version of Theorem 6.3 for general compact connected Lie groups. For this we need some preliminary remarks.

Let $G$ be a compact connected Lie group. Then, the inclusion $T_{G} \subset G$ of a maximal torus of $G$ induces an isomorphism $\pi_{1}\left(T_{G}\right)_{W_{G}} \cong \pi_{1}(G)$. Here, $\pi_{1}\left(T_{G}\right)_{W_{G}}$ denotes the covariants, i.e. the quotient of $\pi_{1}\left(T_{G}\right)$ by the sublattice generated by all elements of the form $a-w(a)$ for $a \in \pi_{1}\left(T_{G}\right)$ and $w \in W_{G}$. This follows from [1, Proposition 5.47] and the detailed description of the kernel of $\pi_{1}\left(T_{G}\right) \rightarrow \pi_{1}(G)$ also given in $\left[1\right.$, Section 5]. Since $\pi_{1}\left(T_{G p} \wedge\right) \cong \pi_{1}\left(T_{G}\right) \otimes \mathbb{Z}_{p}^{\wedge} \cong H_{2}\left(B T ; \mathbb{Z}_{p}^{\wedge}\right) \cong$ $H_{2}\left(B T_{G p} \wedge \mathbb{Z}\right)$ are isomorphisms of $W_{G}$-modules, since $\pi_{1}\left(G_{p}^{\wedge}\right) \cong \pi_{1}(G) \otimes \mathbb{Z}_{p}^{\wedge} \cong$ 
$H_{2}\left(B G ; \mathbb{Z}_{p}^{\wedge}\right) \cong H_{2}\left(B G_{p}^{\wedge} ; \mathbb{Z}_{p}^{\wedge}\right)$, and since passing to covariants obviously commutes with tensoring with $\mathbb{Z}_{p}^{\wedge}$, we get an isomorphism $H_{2}\left(B T_{G p}^{\wedge} ; \mathbb{Z}\right)_{W_{G}} \cong H_{2}\left(B G_{p}^{\wedge} ; \mathbb{Z}\right)$.

Lemma 7.1. Let $G$ be a compact connected Lie group satisfying condition $\left(C_{p}\right)$. Let $X$ be a connected $p$-compact group with the same Weyl group type as $G$. Then the following hold:

1. $\mathrm{H}_{2}(B X ; \mathbb{Z})$ is torsion free.

2. For all $r$, the maps $B T_{G p} \wedge \rightarrow B G_{p}^{\wedge}$ and $B T_{G p} \wedge \rightarrow B X$ induce isomorphisms $H^{2}\left(B X ; \mathbb{Z} / p^{r}\right) \cong H^{2}\left(B T_{G p}^{\wedge} ; \mathbb{Z} / p^{r}\right)^{W_{G}} \cong H^{2}\left(B G_{p}^{\wedge} ; \mathbb{Z} / p^{r}\right)$.

Proof. Let $L:=H_{2}\left(B T_{G p} \wedge \mathbb{Z}\right)$ and let $W:=W_{G}$. Since $G$ satisfies condition $\left(C_{p}\right)$, we have $H^{*}\left(B G_{p}^{\wedge} ; \mathbb{Z}_{p}^{\wedge}\right) \cong H^{*}\left(B T_{G p}^{\wedge} ; \mathbb{Z}_{p}^{\wedge}\right)^{W}$. In particular, $H^{*}\left(B G_{p}^{\wedge} ; \mathbb{Z}_{p}^{\wedge}\right)$ is torsion free, and so is $L_{W} \cong H_{2}\left(B G_{p}^{\wedge} ; \mathbb{Z}_{p}^{\wedge}\right)$.

The maximal torus $B T_{G p} \wedge B X$ of the connected $p$-compact group $X$ induces epimorphisms $L_{W} \rightarrow \pi_{1}(X)$ and $L_{W} \rightarrow H_{2}(B X ; \mathbb{Z})$. The kernel of this map is finite, since rationalizing gives an isomorphism $L_{W} \otimes \mathbb{Q} \cong H_{2}(B X ; \mathbb{Z}) \otimes \mathbb{Q}$. This follows by dualizing the isomorphism of Proposition 2.9 (2) and from the fact that dualizing transforms invariants into covariants. Because $L_{W}$ is torsion free, the kernel is trivial and $L_{W} \rightarrow H_{2}(B X ; \mathbb{Z})$ an isomorphism.

Since $G$ satisfies condition $\left(C_{p}\right)$, we have $H^{*}\left(B G_{p}^{\wedge} ; \mathbb{Z} / p^{r}\right) \cong H^{*}\left(B T_{G_{p}}^{\wedge} ; \mathbb{Z} / p^{r}\right)^{W}$ for all $r$ [15, 4.2]. An application of the universal coefficient theorem now proves the second part.

As mentioned in the introduction, for every compact connected Lie group $G$, there exists a finite covering $1 \rightarrow K \rightarrow \widetilde{G} \stackrel{q}{\rightarrow} G \rightarrow 1$, where $\widetilde{G} \cong G_{s} \times T$ is a product of a simply connected Lie group $G_{s}$ and a torus $T$. The group $K \subset \widetilde{G}$ is a central subgroup. We call this covering universal finite. The group $\widetilde{G}$ is uniquely determined, but not the map $q$. One can compose $q$ with a finite self-covering of $T$.

Every universal finite covering establishes two associated commutative diagrams of exact sequences of compact Lie groups:

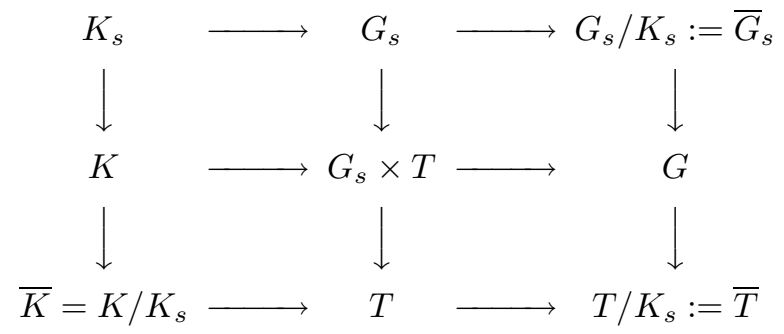

and

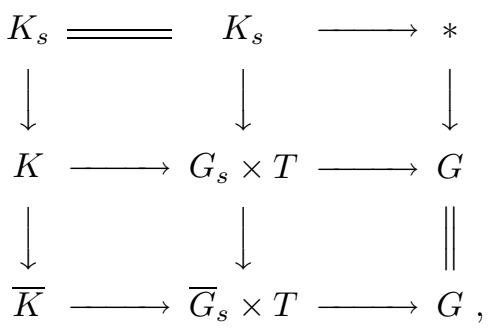

where $K_{s}:=K \cap\left(G_{s} \times\{0\}\right)$. Because $H^{1}\left(\bar{G}_{s} ; \mathbb{Z}\right)=0$, the sequence $\bar{G}_{s} \longrightarrow G \longrightarrow \bar{T}$ induces isomorphisms $H^{1}(\bar{T} ; \mathbb{Z}) \cong H^{1}(G ; \mathbb{Z})$ and $H^{2}(B \bar{T} ; \mathbb{Z}) \cong H^{2}(B G ; \mathbb{Z})$. On the 
other hand, these isomorphisms determine the maps $G \longrightarrow \bar{T}$ and $B G \longrightarrow B \bar{T}$, and therefore also the fibration $B \bar{G}_{s} \longrightarrow B G \longrightarrow B \bar{T}$.

If $G$ satisfies condition $\left(T_{p}\right)$, then, by $[15,4.1], B G_{s} \times B T$ and $B \bar{G}_{s} \times T$ as well as $B G_{s}$ and $B \bar{G}_{s}$ are $p$-torsion free. The order $\left|K_{s}\right|$ of $K_{s}$ is coprime to $p$, because $\pi_{2}\left(B \bar{G}_{s}\right) \cong H_{2}(B \bar{G} ; \mathbb{Z}) \cong K_{s}$.

7.2 Theorem. Let $G$ be a compact connected Lie group satisfying condition $\left(C_{p}\right)$. Let $X$ be a connected $p$-compact group with the same Weyl group type as $G$. Then the following hold:

1. $H^{*}\left(B X ; \mathbb{Z}_{p}^{\wedge}\right) \cong H^{*}\left(B T_{G} ; \mathbb{Z}_{p}^{\wedge}\right)^{W_{G}}$.

2. $B N\left(T_{G}\right)_{p}^{\circ}$ and $B N\left(T_{X}\right)$ are homotopy equivalent.

3. $B X$ and $B G_{p}^{\wedge}$ are homotopy equivalent.

Proof. The finite covering

$$
K \longrightarrow G_{s} \times T \longrightarrow G
$$

$G_{s}$ simply connected and $T$ a torus, establishes a fibration

$$
B K_{p} \longrightarrow B G_{s p}^{\wedge} \times B T_{p}^{\wedge} \longrightarrow B G_{p}^{\wedge},
$$

where $K_{p}$ is the $p$-Sylow subgroup of $K$. This fibration is classified by a map $B G_{p}^{\wedge} \longrightarrow B B K_{p}$. By Lemma 7.1, there is a corresponding map $B X \longrightarrow B B K_{p}$ classifying the fibration

$$
B K_{p} \rightarrow B Y \rightarrow B X .
$$

The total space $B Y$ is simply connected and $p$-complete. Hence, $Y:=\Omega B Y$ is a connected $p$-compact group [13,3.3], and $Y \rightarrow X$ is a finite covering of connected $p$-compact groups. The fibration fits into

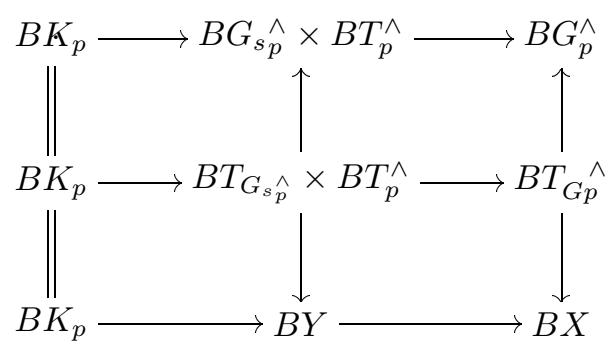

The Weyl group $W_{G}=W_{G_{s}}$ acts as Weyl group on $B G_{s p}^{\wedge} \times B T_{p}^{\wedge} \rightarrow B Y$. Thus, $Y$ has the same $p$-adic Weyl group type as $G_{s p} \times T_{p}^{\wedge}$. By Theorem 6.3, the spaces $B G_{s} \times B T$ and $B Y$ are homotopy equivalent. Moreover, this equivalence fits into a diagram

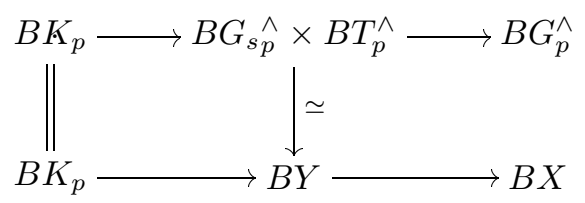

We have to fill in the arrow in the right column. This is done by the following trick. The top row is a principal fibration [3, 7.2]. The composition $B K_{p} \rightarrow B Y \rightarrow B X$ is null homotopic and, because $X$ has finite $\bmod -p$ cohomology, the map $B K_{p} \rightarrow$ $\operatorname{map}\left(B K_{p}, B X\right)_{\text {const }}$ is an equivalence [17]. By a lemma of Zabrodsky [21] (for details see also [3, 9.8 and the proof of 9.7]), we can fill in the arrow in the right 
column so as to make the diagram commutative up to homotopy. This arrow is an equivalence, which is the third statement. The first two statements follow obviously from the third.

Proof of Theorem 1.9. Part (1) implies (3) by Theorem 7.2. The implications from (3) to (2) and from (2) to (1) are obvious.

\section{REFERENCES}

[1] J.F. Adams, Lecturs on Lie groups, W. A. Benjamin, 1969. MR 40:5780

[2] J.F. Adams and Z. Mahmud, Maps between classifying spaces, Invent. Math. 35 (1976), 1-41. MR 54:11331

[3] J. Aguadé, C. Broto and D. Notbohm, Homotopy classification of some spaces with interesting cohomology and a conjecture of Cooke, Part I, Topology 33 (1994), 455-492. MR 95i:55006

[4] A. Bousfield and D. Kan, Homotopy limits, completions and localizations, Lecture Notes in Math. 304, Springer Verlag, 1972. MR 51:1825

[5] W.G. Dwyer and C.W. Wilkerson, Homotopy fixed point methods for Lie groups and finite loop spaces, Ann. of Math. (2) 139 (1994), 395-442. MR 95e:55018

[6] W.G. Dwyer and C.W. Wilkerson, The center of a p-compact group, The Cech Contennial, Contemp. Math. 181 (1995), 119-157. MR 96a:55024

[7] W.G. Dwyer and A. Zabrodsky, Maps between classifying spaces, Proceedings of the 1986 Barcelona Conference on Algebraic Topology, Lecture Notes in Math. 1298, Springer Verlag, 1988, pp. 109-119. MR 89b:55018

[8] J.H. Gunawardena, J. Lannes, and S. Zarati, Cohomlogie des groupes symmétrique et application de Quillen, L.M.S Lectures Note Ser. 139 (1989), 61-68. MR 91d:18013

[9] R.M. Kane, The Homology of Hopf Spaces, North-Holland, 1988. MR 90f:55018

[10] S. MacLane, Homology, Springer Verlag, Berlin Heidelberg New York, 1975. MR 50:2285

[11] J. Lannes, Sur les espaces fonctionnels dont la source est le classifiant d'un p-groupe abélien élémentaire, Publ. Math. IHES 75 (1992), 135-244. MR 93j:55019

[12] J.M. Møller, Rational isomorphisms between p-compact groups, Topology 35 (1996), 201-225. MR 97b:55019

[13] J.M. Møller and D.Notbohm, Centers and finite coverings of finite loop spaces, J. reine u. angew. Math. 456 (1994), 99-133. MR 95j:55029

[14] D. Notbohm, Fake Lie groups with maximal tori IV, Math. Ann. 294 (1992), 109-116. MR 94e:55025

[15] D. Notbohm, Homotopy uniqueness of classifying spaces of compact connected Lie groups at primes dividing the order of the Weyl group, Topology 33 (1994), 271-330. MR 95e:55020

[16] D. Notbohm, On the classifying space functor for compact Lie groups, J. London Math. Soc. 52 (1995), 185-198. MR 96g:55019

[17] D. Notbohm, Kernels of maps between classifying spaces, Israel J. Math. 87 (1994), 243-256. MR 95k:55032

[18] D.Notbohm and L.Smith, Fake Lie groups and maximal tori I, Math. Ann. 288 (1991), 637-661. MR 93k:55012a

[19] D.L. Rector, Loop structures on the homotopy type of $S^{3}$, Symposium on Algebraic Topology, Seattle 1971 (P.J. Hilton, ed.), Lecture Notes in Mathematics 249, Springer-Verlag, BerlinHeidelberg-New York, 1971. MR 49:3916

[20] C. Wilkerson, Rational maximal tori, J. Pure Appl. Algebra 4 (1974), 261-272. MR 49:8008

[21] A. Zabrodsky, On phantom maps and a theorem of H. Miller, Israel J. Math. 58 (1987), 129-143. MR 88m:55028

Matematisk Institut, Universitetsparken 5, DK-2100 København $\varnothing$, Denmark

E-mail address: moller@math.ku.dk

Mathematisches Institut, Bunsenstr. 3-5, 37073 Göttingen, Germany

E-mail address: notbohm@cfgauss.uni-math.gwdg.de 